\title{
Soins intégrés en Suisse
}

\author{
Séverine Schusselé Filliettaz ${ }^{a}$, Peter Berchtold ${ }^{b}$, Dimitri Kohlerc, Isabelle Peytremann-Bridevaux ${ }^{d}$ \\ ${ }^{a}$ Forum Managed Care, Institut de médecine sociale et préventive, Lausanne; ${ }^{b}$ Dr méd., Forum Managed Care, membre FMH; ${ }^{\circ}$ Observatoire suisse de la \\ santé (Obsan); d Dr méd., Institut de médecine sociale et préventive, Lausanne, membre FMH
}

C’est un fait désormais reconnu: une prise en charge de qualité requiert plus de communication, plus de coordination et plus de coopération entre les prestataires de soins. Les résultats de la première Enquête suisse sur les soins intégrés montrent que nombreuses sont les initiatives qui travaillent de cette manière. Comment valoriser ces expériences et contribuer à promouvoir les soins intégrés? Quelles leçons tirer de toutes ces initiatives?

La fragmentation des soins et l'augmentation de la prévalence des maladies chroniques mettent les systèmes de santé sous pression. Pour y répondre, de nombreuses initiatives ont vu le jour en Suisse comme ailleurs: elles visent notamment à renforcer la coordination et l'intégration des soins. En menant l'Enquête suisse sur les soins intégrés, le Forum Managed Care, l'Institut universitaire de médecine sociale et préventive de Lausanne et l'Observatoire suisse de la santé ont souhaité contribuer à ce momentum. Cette enquête avait pour objectif de répertorier de manière détaillée l'ensemble des initiatives ciblant l'intégration des soins. Ses résultats, qui viennent d'être publiés [1], contribueront à une meilleure visibilité des activités de soins intégrés existantes et à susciter le partage d'expériences.

\section{Une enquête menée sur toute la Suisse}

Cette enquête a été menée sur l'ensemble du territoire suisse entre juillet 2015 et juillet 2016. Pour être éligibles, les initiatives devaient répondre aux quatre critères suivants: 1) formalisation des principes d'intégration; 2) intégration/coordination d'au moins deux niveaux de services de santé; 3) participation d'au moins deux groupes de professionnels de santé différents; 4) être en activité au moment de l'enquête.

Afin d'obtenir la plus grande exhaustivité possible, des contacts ont été systématiquement pris auprès des principaux acteurs du système de santé suisse, aussi bien au niveau national qu'au niveau cantonal ou local (prestataires de soins, financeurs, autorités, structures de formation et de recherche, organisations professionnelles et communautaires). Un questionnaire en ligne a ensuite été proposé aux responsables des initiatives éligibles afin de collecter différentes informations concernant le contexte de la prise en charge, les groupes cibles, les professionnels impliqués, les services proposés, les niveaux intégrés, le financement et l'évaluation des initiatives.

Les 853 contacts effectués ont permis d'identifier 172 initiatives de soins intégrés répondant aux critères d'éligibilité. Un taux de réponse au questionnaire de plus de $94 \%$ a permis d'analyser les données de 155 initiatives principales et de 7 sous-programmes. Ces 155 initiatives ont été réparties dans une des six catégories suivantes: les initiatives ciblant avant tout des "Groupes cibles spécifiques» (34\%), celles destinées à des questions de «Santé mentale \& psychiatrie» (26\%), les «Centres de santé», les initiatives centrées sur des activités de «Transition \& coordination» (16\%), les «Réseaux de médecins» (6\%) et enfin les initiatives axées sur les «Médicaments» (5\%).

\section{Un essor et une diversité remarquables}

La mise en œuvre des soins intégrés connaît une accélération depuis quelques années: tandis que la pre-

\section{Symposium national des soins intégrés,}

\section{4 juin 2017, Kursaal Berne}

II y a encore quelques années, les soins intégrés en Suisse se limitaient principalement aux réseaux de médecins de premier recours. Leur contribution reste extrêmement précieuse et, parallèlement, une multitude de nouvelles formes d'intégration des soins sont apparues. Le Symposium du fmc - le forum suisse des soins intégrés aura lieu le 14 juin prochain. Intitulé cette année "Smarter Healthcare - l'art de l'intégration intelligente», il permettra de découvrir et de discuter les développements, les stratégies et les pratiques d'intégration des soins. Les participant-e-s pourront d'ailleurs approfondir cette thématique et partager leurs expertises dans des ateliers ainsi que lors des tablesrondes. Plus d'information sur le Symposium fmc 2017 sous www.fmc.ch. 
mière moitié des initiatives incluses dans l'enquête se sont développées entre 1990 et 2009, la seconde moitié des initiatives identifiées ont été introduites sur six ans, soit entre 2010 et 2016. Jusqu'en 2012, la majorité des initiatives se trouvaient en Suisse alémanique. Aujourd'hui, 52\% d'entre elles se trouvent en Suisse latine, $45 \%$ en Suisse alémanique, tandis que $3 \%$ sont actives dans les deux régions linguistiques. Les types d'initiatives diffèrent également entre les régions: les «Réseaux de médecins» et les "Centres de santé» sont plus nombreux en Suisse alémanique, tandis que les initiatives axées sur les "Groupes cibles spécifiques» ou sur les "Médicaments», ainsi que les initiatives de "Transition \& coordination" sont plus fréquentes dans la partie latine du pays.

Il est également intéressant de noter l'évolution chronologique de la mise en œuvre des initiatives des différentes catégories (fig. 1). L'augmentation est la plus marquée pour les initiatives visant des «Groupes cibles spécifiques» et pour celles de «Santé mentale \& psychiatrie», des problématiques généralement chroniques et qui requièrent une prise en charge coordonnée. A noter également l'émergence depuis 2008 d'initiatives qui ne

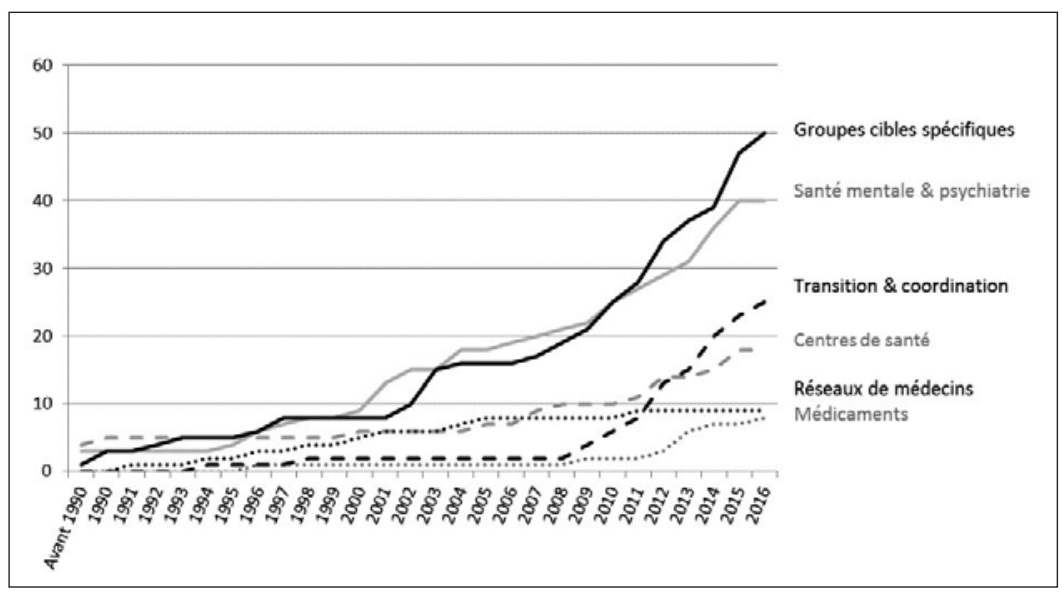

Figure 1: Nombre cumulé d'initiatives de soins intégrés, par catégorie, Suisse 1990-2016. ciblent pas de problématique spécifique mais qui s'intéressent aux aspects génériques de «Transition \& coordination".

Les initiatives de soins intégrés se caractérisent également par l'implication d'un nombre important de groupes professionnels: les initiatives incluent en moyenne cinq groupes de professionnels de santé. Cependant, sept groupes professionnels sont impliqués dans les "Centres de santé», suivis par les initiatives visant les «Groupes cibles spécifiques» $(n=6)$ puis par celles de «Transition \& coordination» $(n=5)$. Les autres catégories impliquent entre quatre et trois groupes professionnels. Les initiatives diffèrent également sous d'autres aspects, dont le nombre de niveaux de santé intégrés: ce sont ainsi les «Centres de santé» qui intègrent le plus de niveaux, tandis que les initiatives ciblant les «Médicaments» en ciblent le moins.

\section{Conclusion}

L'Enquête suisse sur les soins intégrés fournit la première cartographie systématique des initiatives menées dans ce domaine dans notre pays. Malgré une organisation fédéraliste du système de santé - a priori peu propice à l'intégration des soins - et malgré des incitatifs limités pour le développement des soins intégrés, les initiatives identifiées sont remarquablement nombreuses, diversifiées et réparties dans toute la Suisse. Les résultats de cette enquête reflètent également un essor des soins intégrés ainsi que des développements différenciés et adaptés aux spécificités des contextes et aux besoins des groupes ciblés par les initiatives.

\section{Crédit illustration}

@ Séverine Schusselé Filliettaz

\section{Référence}

1 Schusselé Filliettaz S, Kohler D, Berchtold P, Peytremann-Bridevaux I (2017). Soins intégrés en Suisse. Résultats de la $1^{\text {re }}$ enquête (2015-2016) (Obsan Dossier 57). Neuchâtel: Observatoire suisse de la santé. www.obsan.ch 